\title{
Prognostic value of serum hyponatremia for outcomes in patients with heart failure with preserved ejection fraction: An observational cohort study
}

\author{
YANG SU $^{1 *}$, MENGQIU MA ${ }^{1 *}$, HENGBIN ZHANG $^{1 *}$, XIN PAN $^{2}$, XIANLING ZHANG ${ }^{1}$, \\ FENGLEI ZHANG ${ }^{3}$, YANGBO $\mathrm{LV}^{1,3}$ and $\mathrm{CHUNXI} \mathrm{YAN}^{1}$ \\ ${ }^{1}$ Department of Cardiology, Shanghai Tenth People's Hospital, Qidong People's Hospital; \\ ${ }^{2}$ Department of Geriatrics, Shanghai Tenth People's Hospital, Tongji University School of Medicine, Shanghai 200072; \\ ${ }^{3}$ Department of Cardiology, Shanghai Yoda Cardiothoracic Hospital, Shanghai 200012, P.R China
}

Received March 9, 2020; Accepted July 1, 2020

DOI: 10.3892/etm.2020.9231

\begin{abstract}
Hyponatremia is a risk factor associated with poor prognosis in patients with heart failure (HF) with reduced ejection fraction. However, whether hyponatremia has a similar role in patients with HF with preserved ejection fraction $(\mathrm{HFpEF})$ has remained controversial. Therefore, the present study aimed to investigate the clinical characteristics and 24-month prognostic profile of a cohort of patients with HFpEF in China. From a registered observational cohort study on 1,027 subjects with HF, 496 patients with HFpEF were included. The association between baseline hyponatremia on admission and 24-month adverse outcomes (including all-cause mortality, re-hospitalization for $\mathrm{HF}$ and stroke) was analyzed using logistic regression with the Cox proportional hazards model. Of the 496 patients with HFpEF with a mean age of 72.8 years and proportion of males of $53.0 \%$, 71 patients were diagnosed with hyponatremia. Furthermore, 29 patients $(5.8 \%)$ were lost to follow-up. The hyponatremia group had lower blood pressure and serum hemoglobin, higher N-terminal pro B-type natriuretic peptide (NT-proBNP) and D-dimer, more patients with a history of atrial fibrillation and a higher proportion of spironolactone and loop diuretic use. According to a multivariate regression analysis, New York Heart Association functional classes III-IV and a serum NT-proBNP level above the median were risk factors
\end{abstract}

Correspondence to: Dr Chunxi Yan or Dr Yangbo Lv, Department of Cardiology, Shanghai Tenth People's Hospital, Qidong People's Hospital, Tongji University School of Medicine, 516 Middle Jianghai Road, Shanghai 200072, P.R. China

E-mail: 18906285119@189.cn

E-mail: blacambb@163.com

*Contributed equally

Key words: hyponatremia, heart failure with preserved ejection fraction, prognosis for hyponatremia, while higher systolic blood pressure and $\beta$-blocker use were protective factors against hyponatremia. In the Kaplan-Meier analysis, hyponatremia was associated with all-causes of mortality, re-hospitalization for HF and a poor prognosis for patients suffering from strokes $(\log$-rank $\mathrm{P}<0.05$ for all 3 endpoints). On multivariate logistic regression analysis with the Cox proportional hazard model, hyponatremia was an independent predictor of three adverse outcomes [all-cause mortality: Hazard ratio $(\mathrm{HR})=1.54,95 \% \mathrm{CI}=1.07-2.91$, $\mathrm{P}=0.034$; re-hospitalization for heart failure: $\mathrm{HR}=1.28,95 \%$ $\mathrm{CI}=1.16-2.47, \mathrm{P}=0.013$; stroke: $\mathrm{HR}=1.78,95 \% \mathrm{CI}=1.04-2.89$, $\mathrm{P}=0.016]$. Collectively, the present results suggested that hyponatremia on admission was significantly associated with all-cause mortality, re-hospitalization and stroke within 24 months in a cohort of hospitalized patients with HFpEF in China. Thus, hyponatremia should be carefully monitored and frequently adjusted in patients with HFpEF (NCT04062500).

\section{Introduction}

Heart failure (HF) is a clinical syndrome associated with poor quality of life, substantial health-care resource utilization and premature mortality (1). Despite the fact that the division of HF with preserved ejection fraction (HFpEF) varies depending on the cut-off point of the left ventricular ejection fraction (LVEF) used, previous studies have reported that HFpEF accounted for $\sim 50 \%$ of patients with HF (2). Unlike HF with reduced $\mathrm{EF}(\mathrm{HFrEF}), \mathrm{HFpEF}$ is characterized by heterogenetic pathophysiology processes and various co-morbidities (3).

Hyponatremia, defined as a serum sodium levels of $<135 \mathrm{mmol} / 1$, is one of the major electrolyte disorders in acute hospitalized patients (4), and its prevalence in patients with acute $\mathrm{HF}$ (AHF) was estimated to be $\leq 25 \%$ (5-7). HF involves fluid retention in the body and diuretics are normally used to induce a negative balance of sodium in patients with $\mathrm{HF}$, and all of this may lead to hyponatremia. A lower serum sodium level in AHF may indicate poor water excretion attributed to cardio-renal insufficiency, which is linked to a worse clinical outcome. Previous studies have revealed that hyponatremia is an independent predictor of mortality and re-hospitalization 
for patients with $\operatorname{HFrEF}(7,8)$ and has been demonstrated in HFpEF populations $(9,10)$. However, there is limited data on the burden of hyponatremia and its complications in patients with HFpEF in China and any other Asian countries.

To the best of our knowledge, the prevalence and factors associated with hyponatremia among patients with $\mathrm{HFpEF}$ have not been studied in China or other Asian countries. Therefore, the aim of the present study was to examine the clinical characteristics of subjects with hyponatremia via a prospective observational study with a cohort of patients with HFpEF and examine the prognostic value regarding adverse outcomes.

\section{Materials and methods}

Study population. In the present prospective observational study, consecutive symptomatic patients with HF hospitalized for the treatment of decompensated HFpEF from Shanghai 10th People's Hospital, Tongji University School of Medicine (Shanghai, China) between July 2017 and December 2018 were enrolled. The study protocol was approved by the Ethics Committee of Shanghai 10th People's Hospital (Shanghai, China). Written informed consent for medical treatment was provided by each patient on admission. The present study was based on a previously registered clinical trial (NCT04062500). Symptomatic HFpEF was defined based on the Framingham criteria (11) and a left ventricular ejection fraction (LVEF) of $>50 \%$. In line with previous studies, patients were $\geq 18$ years of age and met the Framingham criteria for the diagnosis of HF (presence of either two major criteria or combination of one major criterion and two minor criteria). Patients with severe liver disease, trauma, infection and recent surgery were excluded. A flow diagram depicting the movement of the patients throughout the study is presented in Fig. 1.

Blood samples and echocardiography were obtained within $24 \mathrm{~h}$ of hospitalization of admission before any treatment was given. Echocardiographic parameters were measured by experienced echocardiographic cardiologists according to the recommendations of the American Society of Echocardiography (12). The left atrial diameter, measured as the diameter from the anterior to the posterior side of the left atrium, the left ventricular end-systolic diameter and the left ventricular end-diastolic diameter were determined in the parasternal long-axis view. The left ventricular ejection fraction was calculated using a biplane methods of disc (modified Simpson's rule) in four-chamber view (13).

The patients' medical history and current medical treatment of angiotensin-converting enzyme inhibitors (ACEI), angiotensin receptor blocker, $\beta$-blockers, aldosterone antagonists, loop diuretics, antiplatelet agents, oral anticoagulants and calcium channel blockers were collected. Cardiac function was evaluated using the New York Heart Association (NYHA) classification (1).

Data preparation and outcome measures. Patients were categorized depending on their level of sodium on first admission to the center. Each patient was assigned to either the hyponatremia group if serum sodium was $<135 \mathrm{mmol} / \mathrm{l}$ or the non-hyponatremia group if sodium was $\geq 135 \mathrm{mmol} / \mathrm{l}$. The follow-up period of the patients was 24 months. Clinical events, including all-cause mortality, re-hospitalization for $\mathrm{HF}$ and stroke, were recorded via telephone enquiry and searching of the patients' electronic medical records. The prognostic value of hyponatremia for the outcomes of patients with HFpEF was investigated.

Statistical analysis. Continuous, normally distributed variables are presented as the mean \pm standard deviation and non-normally distributed data as the median (interquartile range). Data were compared using the independent-samples Student's t-test or by one-way analysis of variance and the Mann-Whitney U-test, as appropriate. Categorical data are presented as n (\%) and compared using Pearson's $\chi^{2}$-test.

Univariate logistic regression analysis was used to assess the influence of relevant variables on hyponatremia that were derived from a comparison of baseline characteristics and published literature (14). In addition, multivariate logistic regression analysis was performed to determine whether they have a significant influence on the outcomes. Cumulative survival rates were calculated according to the Kaplan-Meier method with the log-rank test used for comparison between hyponatremia and non-hyponatremia groups, setting statistical power by assuming an event rate of 0.3 for all-cause mortality, re-hospitalization for $\mathrm{HF}$ and stroke.

Cox-proportional hazards models were used to calculate the hazard ratio (HR) with corresponding 95\% CI for occurrence of the primary study end-points (all-cause mortality, re-hospitalization for HF and stroke) associated with each pattern of hyponatremia development. All-cause mortality was adjusted by age, sex, body mass index (BMI), systolic blood pressure (SBP), hyponatremia, N-terminal pro B-type natriuretic peptide (NT-proBNP), NYHA class, atrial fibrillation history, estimated glomerular filtration rate (eGFR) and hemoglobin. Re-hospitalization for HF was adjusted by age, sex, BMI, SBP, hyponatremia, NT-proBNP, NYHA class, atrial fibrillation history, eGFR and hemoglobin. Furthermore, stroke was adjusted by age, sex, SBP, hyponatremia, D-dimer, atrial fibrillation history, anti-platelet use and statin use.

$\mathrm{P}<0.05$ was considered to indicate statistical significance. All statistical analyses were performed using SPSS v.25 (IBM Corp.).

\section{Results}

Baseline clinical characteristics of patients with HFpEF. In

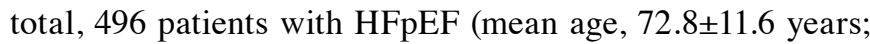
$53.0 \%$ males) were included and the mean serum sodium level was $140.1 \pm 4.7 \mathrm{mmol} / 1$. Patients with hyponatremia had a mean sodium level of $131.1 \pm 3.6 \mathrm{mmol} / \mathrm{l}$. Baseline characteristics of patients with HFpEF are presented in Table I. The hyponatremia group had a significantly lower blood pressure (SBP, $112.1 \pm 13.1$ vs. $137.1 \pm 22.5 \mathrm{mmHg}, \mathrm{P}<0.001$; diastolic blood pressure (DBP), $67.7 \pm 11.6$ vs. $76.9 \pm 12.4 \mathrm{mmHg}, \mathrm{P}=0.002)$ and hemoglobin levels $(113.4 \pm 14.4$ vs. $123.2 \pm 22.1 \mathrm{~g} / 1, \mathrm{P}<0.001)$ but higher NT-proBNP [1,168.0 (586.1-3,000.0) vs. 1,046.0 (525.8-2,380.5), $\mathrm{P}<0.001]$ and $\mathrm{D}$-dimer levels [0.78 (0.48-1.43) vs. $0.47(0.27-0.90), \mathrm{P}<0.001]$. Furthermore, the hyponatremia group contained a larger proportion of patients with a history of atrial fibrillation (49.3 vs. $28.9 \%, \mathrm{P}<0.001)$. The proportion of patients taking aldosterone antagonists and loop diuretics 


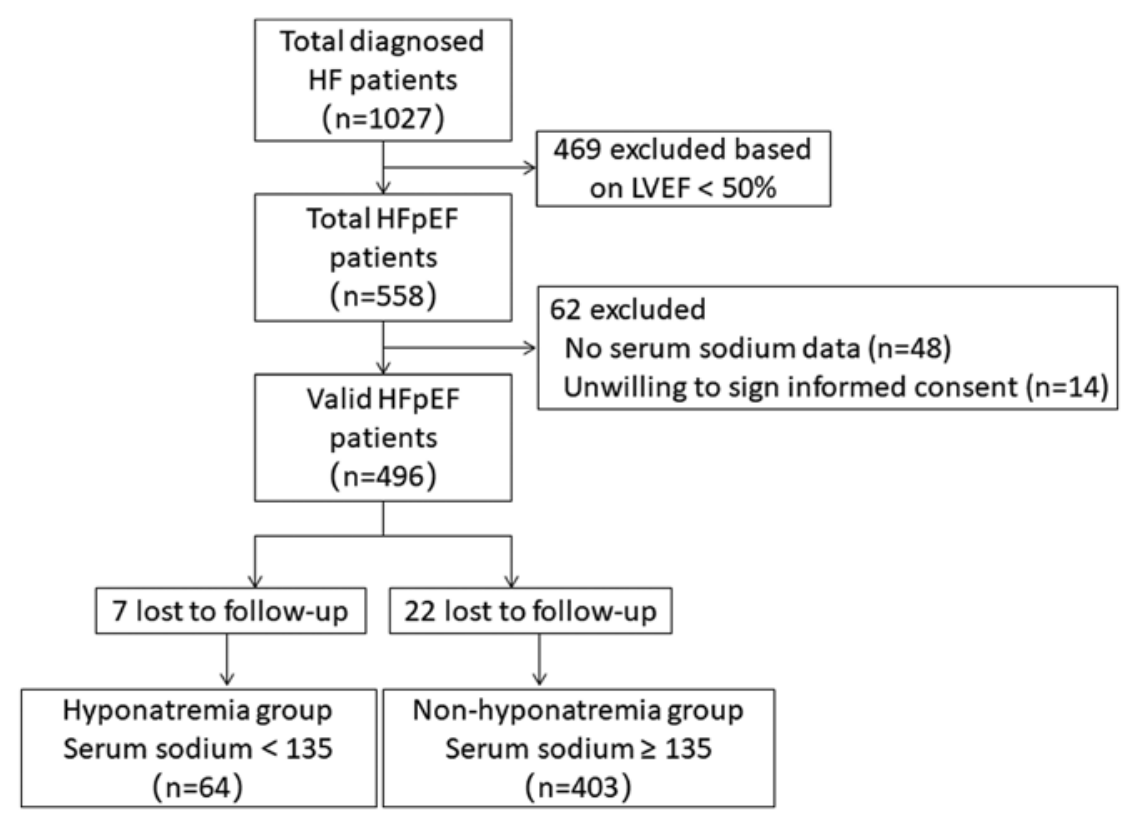

Figure 1. Flow chart of the study. LVEF, left ventricle ejection fraction; HF, heart failure; HFpEF, HF with preserved ejection fraction.

was higher in the hyponatremia group (59.2 vs. $33.4 \%$, $\mathrm{P}<0.001 ; 66.2$ vs. $40.2 \%, \mathrm{P}<0.001$, respectively). Furthermore, there were no differences in age, sex, percentage of NYHA III-IV, eGFR, lipid profile, blood glucose, echocardiographic parameters and other medications that the patients were taking.

Serum sodium distribution of patients with HFpEF and logistic regression analysis for risk factors of hyponatremia. The distribution of serum sodium levels was analyzed in patients with $\mathrm{HFpEF}$ and the majority of patients was within the interval of 141.5-142.4 mmol/l ( $\mathrm{n}=56$; Fig. S1).

Univariate logistic regression analyses identified that hyponatremia was positively associated with NT-proBNP $>$ median [odds ratio $(\mathrm{OR})=6.045,95 \% \mathrm{CI}=3.41-10.72]$, NYHA classification III-IV (OR=2.994, 95\% CI=1.714-5.057), atrial fibrillation history ( $\mathrm{OR}=2.100,95 \% \mathrm{CI}=1.233-3.577)$, loop diuretics ( $\mathrm{OR}=1.875,95 \% \mathrm{CI}=1.094-3.215)$ and spironolactone $(\mathrm{OR}=1.799,95 \% \mathrm{CI}=1.058-3.058)$, but it was negatively associated with $\beta$-blockers $(\mathrm{OR}=0.569,95 \% \mathrm{CI}=0.331-0.981), \mathrm{SBP}$ $(\mathrm{OR}=0.946,95 \% \mathrm{CI}=0.930-0.962)$ and $\mathrm{DBP}(\mathrm{OR}=0.965$, 95\% CI=0.945-0.986). Multivariate logistic regression analysis demonstrated that in patients with HFpEF, a higher NT-proBNP (above median, OR=4.521, 95\% CI=2.450-9.584) or worse cardiac function $(\mathrm{OR}=2.275,95 \% \mathrm{CI}=1.207-4.338)$ were positively associated with hyponatremia. In addition, a higher SBP $(\mathrm{OR}=0.942,95 \%$ CI $0.920-0.964)$ and $\beta$-blocker use $(\mathrm{OR}=0.343,95 \% \mathrm{CI}=0.186-0.675)$ were negatively associated with hyponatremia (Table II).

Clinical outcomes of patients with HFpEF. In total, 29 (6\%) patients were lost to follow-up, mortality occurred in 71 (15.2\%) cases in the first 24 months, 129 (27.6\%) patients were re-hospitalized for HF and 77 (16.5\%) patients had a stroke. Furthermore, the hyponatremia group had a worse prognosis compared with the non-hyponatremia group. All-cause mortality was $39.1 \%$ in the hyponatremia group compared with $11.4 \%$ in the non-hyponatremia group $(\mathrm{P}<0.001)$. In the hyponatremia group, $40.6 \%$ of patients were re-hospitalized for $\mathrm{HF}$, as compared with $25.6 \%$ in the non-hyponatremia group $(\mathrm{P}=0.016)$. Furthermore, the prevalence of stroke was $28.1 \%$ in the hyponatremia group as compared with $14.6 \%$ in the non-hyponatremia group $(\mathrm{P}=0.011$; Table III).

Survival analysis. The median duration of the follow up of the study participants was 24 months. The number of mortalities in the hyponatremia group was 25 (39.1\% of patients) compared with 46 in the non-hyponatremia group (11.4\% of patients). Kaplan-Meier survival curves (Fig. 2) indicated that there were significant differences in survival status, re-hospitalization and stroke between the two groups of patients with HFpEF. In addition, patients with sodium levels $\geq 135 \mathrm{mmol} / 1$ had an improved prognosis compared with patients with hyponatremia.

In order to investigate the predictors of adverse events in patients with HFpEF, a multivariate Cox proportion hazard regression model was established for all-cause mortality, re-hospitalization and stroke (Fig. 3). It was indicated that hyponatremia was an independent predictor for three adverse events in patients with HFpEF (all-cause mortality: Adjusted $\mathrm{HR}=1.54,95 \% \mathrm{CI}=1.07-2.91, \mathrm{P}=0.034$; re-hospitalization: Adjusted HR=1.28, 95\% CI=1.16-2.47, $\mathrm{P}=0.013$; stroke: Adjusted HR=1.78, 95\% CI=1.04-2.89, $\mathrm{P}=0.016$ ). Another independent predictor for all-cause mortality was older age (adjusted HR=1.07, 95\% CI=1.05-1.09, $\mathrm{P}<0.001$ ). Furthermore, lower SBP (adjusted HR=0.97, 95\% CI $=0.97-0.98$, $\mathrm{P}<0.001$ ), NT-proBNP $>$ median (adjusted $\mathrm{HR}=1.62,95 \%$ $\mathrm{CI}=1.05-3.03, \mathrm{P}=0.027$ ), NYHA III-IV (adjusted HR=1.73, 95\% $\mathrm{CI}=1.07-2.81, \mathrm{P}=0.021$ ), AF history (adjusted $\mathrm{HR}=2.69$, 95\% $\mathrm{CI}=1.32-3.58, \mathrm{P}<0.001)$ and $\mathrm{eGFR}<60 \mathrm{ml} / \mathrm{min} / 1.73 \mathrm{~m}^{2}$ (adjusted HR=0.51, 95\% CI=0.28-0.91, P=0.024) were significantly associated with all-cause mortality. Other independent predictors for re-hospitalization were older age (adjusted $\mathrm{HR}=1.04,95 \% \mathrm{CI}=1.02-1.07, \mathrm{P}=0.008$ ), lower SBP (adjusted $\mathrm{HR}=0.96,95 \% \mathrm{CI}=0.95-0.99, \mathrm{P}=0.001)$, NT-proBNP $>$ median 
Table I. Baseline clinical characteristics of patients with heart failure with preserved ejection fraction based on serum sodium status.

\begin{tabular}{|c|c|c|c|c|}
\hline \multirow[b]{2}{*}{ Item } & \multirow[b]{2}{*}{ Normal range } & \multicolumn{2}{|c|}{ Serum sodium concentration $(\mathrm{mmol} / \mathrm{l})$} & \multirow[b]{2}{*}{ P-value } \\
\hline & & $<135(\mathrm{n}=71)$ & $\geq 135(n=425)$ & \\
\hline Age (years) & - & $73.9 \pm 11.8$ & $72.6 \pm 11.6$ & 0.381 \\
\hline Male sex & - & $31(43.7)$ & $232(54.6)$ & 0.104 \\
\hline $\operatorname{BMI}\left(\mathrm{kg} / \mathrm{m}^{2}\right)$ & - & $24.1 \pm 3.80$ & $23.9 \pm 4.16$ & 0.342 \\
\hline $\mathrm{SBP}(\mathrm{mmHg})$ & - & $112.1 \pm 13.1$ & $137.1 \pm 22.5$ & $<0.001$ \\
\hline $\mathrm{DBP}(\mathrm{mmHg})$ & - & $67.7 \pm 11.6$ & $76.9 \pm 12.4$ & 0.002 \\
\hline Heart rate (beats/min) & - & $88.3 \pm 17.3$ & $89.9 \pm 17.5$ & 0.490 \\
\hline NYHA class III-IV & - & $65(91.5)$ & $391(92.0)$ & 0.761 \\
\hline \multicolumn{5}{|l|}{ Medical history } \\
\hline Diabetes & - & $24(33.8)$ & $153(36.0)$ & 0.790 \\
\hline Hypertension & - & $48(67.6)$ & $292(68.7)$ & 0.893 \\
\hline Coronary artery disease & - & $43(60.6)$ & $256(60.2)$ & 1.000 \\
\hline Atrial fibrillation & - & $35(49.3)$ & $123(28.9)$ & $<0.001$ \\
\hline \multicolumn{5}{|l|}{ Laboratory data } \\
\hline Hemoglobin $(\mathrm{g} / \mathrm{l})$ & $110-160$ & $113.4 \pm 14.4$ & $123.2 \pm 22.1$ & $<0.001$ \\
\hline CRP (mg/l) & $0-10$ & $7.65(3.02-18.0)$ & $3.23(3.02-11.9)$ & 0.079 \\
\hline Alb (g/l) & $35-50$ & $38.3 \pm 5.60$ & $39.7 \pm 4.81$ & 0.082 \\
\hline $\mathrm{UA}(\mu \mathrm{mol} / \mathrm{l})$ & $<420$ & $371.6 \pm 73.5$ & $383.1 \pm 62.7$ & 0.523 \\
\hline eGFR (ml/min/1.73 m²) & $>90$ & $69.9 \pm 23.9$ & $67.1 \pm 23.5$ & 0.357 \\
\hline $\mathrm{TC}(\mathrm{mmol} / \mathrm{l})$ & $2.8-5.2$ & $3.75 \pm 1.04$ & $3.78 \pm 1.14$ & 0.831 \\
\hline LDL-C (mmol/l) & $<3.1$ & $1.98 \pm 0.86$ & $2.08 \pm 1.00$ & 0.442 \\
\hline HDL-C (mmol/l) & $0.8-1.8$ & $1.09 \pm 0.39$ & $1.14 \pm 0.65$ & 0.698 \\
\hline Sodium $(\mathrm{mmol} / \mathrm{l})$ & $135-145$ & $131.1 \pm 3.6$ & $141.6 \pm 2.8$ & $<0.001$ \\
\hline Potassium (mmol/l) & $3.5-5.5$ & $4.19 \pm 0.53$ & $3.98 \pm 0.54$ & 0.980 \\
\hline $\operatorname{HbA1C}(\%)$ & $4-6$ & $6.42 \pm 1.92$ & $6.39 \pm 1.60$ & 0.195 \\
\hline NT-proBNP (ng/l) & $\begin{array}{l}125 \text { ( }<75 \text { years }) \\
450(\geq 75 \text { years })\end{array}$ & $1,168.0(586.1-3,000.0)$ & $1,046.0(525.8-2,380.5)$ & $<0.001$ \\
\hline D-dimer (mg/l) & $<0.55$ & $0.78(0.48-1.43)$ & $0.47(0.27-0.90)$ & $<0.001$ \\
\hline \multicolumn{5}{|c|}{ Echocardiographic parameters } \\
\hline $\operatorname{LVEF}(\%)$ & - & $58.1 \pm 4.35$ & $58.9 \pm 4.45$ & 0.104 \\
\hline $\mathrm{LAD}(\mathrm{mm})$ & - & $41.0 \pm 6.12$ & $41.1 \pm 6.62$ & 0.690 \\
\hline LVeDD (mm) & - & $45.4 \pm 4.88$ & $45.9 \pm 4.99$ & 0.512 \\
\hline Levs (mm) & - & $29.7 \pm 4.86$ & $29.9 \pm 4.80$ & 0.744 \\
\hline \multicolumn{5}{|l|}{ Medications } \\
\hline ACEI/ARB & - & $29(40.8)$ & $170(40.1)$ & 0.847 \\
\hline Beta-blocker & - & $48(67.7)$ & $302(71.1)$ & 0.059 \\
\hline Aldosterone blocker & - & $42(59.2)$ & $142(33.4)$ & $<0.001$ \\
\hline Loop diuretic & - & $47(66.2)$ & $171(40.2)$ & $<0.001$ \\
\hline Antiplatelet agent & - & $55(77.5)$ & 345 (81.2) & 0.519 \\
\hline Oral anticoagulant & - & $26(36.6)$ & $189(44.5)$ & 0.248 \\
\hline Calcium channel blocker & - & $22(31.0)$ & $144(33.9)$ & 0.692 \\
\hline Cost (Yuan) & - & $31,140.1 \pm 30,503.2$ & $32,583 \pm 64,318.6$ & 0.771 \\
\hline
\end{tabular}

Continuous variables are presented as the mean \pm standard deviation if they conform to a normal distribution, and otherwise as the median with interquartile range. Categorical variables are presented as $\mathrm{n}(\%)$. Cost refers to the cost of medical treatment during hospitalized period. BMI, body mass index; SBP, systolic blood pressure; DBP, diastolic blood pressure; CRP, c-reactive protein; Alb, albumin; UA, uric acid; eGFR, estimated glomerular filtration rate; TC, total cholesterol; LDL-C, low-density lipoprotein cholesterol; HDL-C, high-density lipoprotein cholesterol; HbA1C, glycated hemoglobin A1C; LVEF, left ventricular ejection fraction; LAD, left atrial diameter; Levs, left ventricular end-systolic diameter; LVeDD, left ventricular end-diastolic diameter; ACEI, angiotensin-converting enzyme inhibitor; ARB, angiotensin receptor blocker; NT-proBNP, N-terminal pro B-type natriuretic peptide; NYHA, New York Heart Association. 
Table II. Univariate and multivariate logistic regression analysis for the risk factors of hyponatremia.

\begin{tabular}{|c|c|c|c|c|c|c|}
\hline \multirow[b]{2}{*}{ Variable } & \multicolumn{3}{|c|}{ Univariate logistic regression } & \multicolumn{3}{|c|}{ Multivariate logistic regression } \\
\hline & OR & $95 \% \mathrm{CI}$ & P-value & OR & $95 \% \mathrm{CI}$ & P-value \\
\hline SBP & 0.946 & $0.930-0.962$ & $<0.001$ & 0.942 & $0.920-0.964$ & $<0.001$ \\
\hline NYHA III-IV & 2.994 & $1.714-5.057$ & $<0.001$ & 2.275 & $1.207-4.338$ & 0.013 \\
\hline Atrial fibrillation history & 2.100 & $1.233-3.577$ & 0.006 & 1.831 & $0.963-3.325$ & 0.057 \\
\hline Beta-blocker & 0.569 & $0.331-0.981$ & 0.043 & 0.343 & $0.186-0.675$ & 0.002 \\
\hline Loop diuretic & 1.875 & $1.094-3.215$ & 0.022 & 1.003 & $0.476-2.235$ & 0.985 \\
\hline Spironolactone & 1.799 & $1.058-3.058$ & 0.030 & 0.907 & $0.422-2.062$ & 0.840 \\
\hline NT-proBNP $>$ median $^{a}$ & 6.045 & $3.41-10.72$ & $<0.001$ & 4.521 & $2.450-9.584$ & 0.001 \\
\hline
\end{tabular}

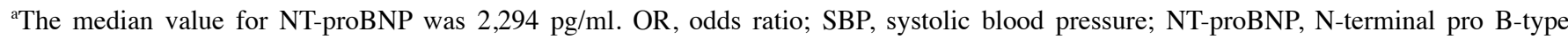
natriuretic peptide; NYHA, New York Heart Association.

Table III. Outcomes of patients with HF with preserved ejection fraction based on serum sodium status.

\begin{tabular}{lrrrr}
\hline & & \multicolumn{2}{c}{ Serum sodium concentration $(\mathrm{mmol} / \mathrm{l})$} & \\
\cline { 4 - 5 } Variable & All patients & $<135(\mathrm{n}=64)$ & $\geq 135(\mathrm{n}=403)$ & P-value \\
\hline All-cause mortality & $71(15.2)$ & $25(39.1)$ & $46(11.4)$ & $<0.001$ \\
Re-hospitalization for HF & $129(27.6)$ & $26(40.6)$ & $103(25.6)$ & 0.016 \\
Stroke & $77(16.5)$ & $18(28.1)$ & $59(14.6)$ & 0.011 \\
\hline
\end{tabular}

P-values were adjusted for age and sex. HF, heart failure.

(adjusted HR=1.24, 95\% $\mathrm{CI}=1.03-2.08, \mathrm{P}=0.042$ ), NYHA III-IV (adjusted HR=2.15, 95\% CI=2.02-2.46, $\mathrm{P}=0.001$ ) and additional AF history (adjusted $\mathrm{HR}=1.48,95 \% \mathrm{CI}=1.05-2.20$, $\mathrm{P}=0.003)$. In addition, other independent predictors for stroke were lower SBP $(\mathrm{HR}=0.99,95 \% \mathrm{CI}=0.98-0.99, \mathrm{P}=0.042)$, higher $\mathrm{D}$-dimer levels $(\mathrm{HR}=1.96,95 \% \mathrm{CI}=1.30-2.59, \mathrm{P}=0.025)$ and atrial fibrillation history $(\mathrm{HR}=1.34,95 \% \mathrm{CI}=1.15-2.47$, $\mathrm{P}=0.018)$.

\section{Discussion}

In the present observational study, it was indicated that hyponatremia was associated with higher all-cause mortality and re-hospitalization for $\mathrm{HF}$ in a cohort of patients with HFpEF; these results were consistent with those of previous studies (14-16). Furthermore, patients with HFpEF with hyponatremia had lower blood pressure, higher serum levels of NT-proBNP and D-dimer, serious anemia and atrial fibrillation, as well as higher rate of medication used, such as spironolactone and loop diuretics, which suggested that the disease in those patients was more serious and should be actively treated. To the best of our knowledge, the present study was the first to demonstrate that hyponatremia was an independent predictor of the incidence of stroke in a patient population with $\mathrm{HFpEF}$.

HFpEF is an increasingly prevalent phenotype of HF. Unlike those for HFrEF, the diagnosis and treatment of HFpEF are not well-standardized, rendering numerous patients with HFpEF misdiagnosed or underdiagnosed. In this situation, the disease may further progress due to poor appetite and low-sodium diet (insufficient intake), and a high dose of diuretic agents (excessive loss), leading to electrolyte disorders, particularly hyponatremia (16).

Hyponatremia is the most common electrolyte disorder (17) and is frequently encountered in patients with HF. It may either arise from depletion (excessive sodium depletion), which is caused by extensive administration of diuretics as aforementioned, or dilution, which is caused by impaired glomerular filtration. Therefore, dilutional hyponatremia in patients with HF with impaired renal function likely results in volume overload, which deteriorates the condition and subsequently leads to a worse prognosis (16). Thus, hyponatremia is an adverse marker of a significant underlying disease and may enhance the severity and complexity of HF. The present results suggested a high incidence of hyponatremia of $14.3 \%$ in hospitalized patients with HF, which is comparable to that reported by other studies on acute decompensated HF $(7,18,19)$.

The causes of hyponatremia are miscellaneous, as the present results suggested that a higher NYHA class and NT-proBNP levels increase the risk, while usage of renin-angiotensin-aldosterone system (RAAS) inhibitor and $\beta$-blockers reduce the risk. On one hand, it has been reported that NYHA class and NT-proBNP correlate with the severity of HF (14). While patients with severe HF require a large dosage of diuretics, this will lead to disturbance of homeostasis and pose a higher risk for hyponatremia. The present results support this concept 


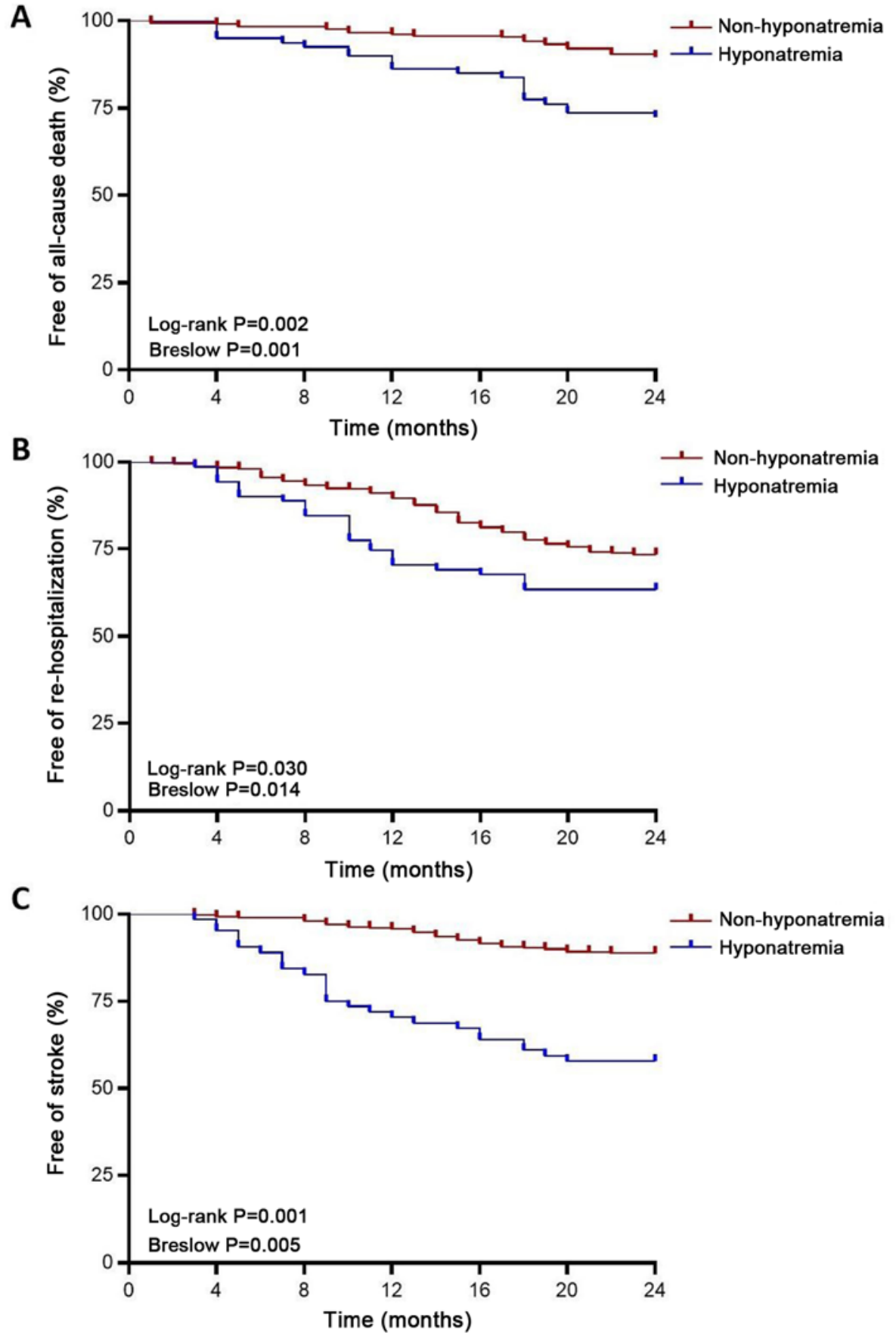

Figure 2. Kaplan Meier survival curves for patients with HFpEF based on the serum sodium status. (A) Rate of survival (no mortality from any cause) in the two groups. (B) Rate of freedom from re-hospitalization in the two groups. (C) Rate of freedom from stroke in the two groups. Patients with hyponatremia had a significantly higher all-cause mortality, re-hospitalization rate and stroke rate $(\mathrm{P}=0.002,0.030$ and 0.001 , respectively). HFpEF, heart failure with preserved ejection fraction.

and are consistent with those of previous studies (20). One the other hand, dilutional hyponatremia resulting from concomitant activation of the RAAS and sympathetic nervous system may be counteracted by the use of RAAS inhibitor or $\beta$-blockers $(21,22)$. This is also in line with the present results.

Previous studies have reported that hyponatremia was associated with worse short-term, mid-term and long-term outcomes $(1,19,23)$. In the OPTIMIZE-HF (24) and OPTIME-CHF (19) trials, following a drop in serum sodium levels (per $3 \mathrm{mmol} / \mathrm{l}$ decrease from $140 \mathrm{mmol} / \mathrm{l}$ ), the OR of in-hospital mortality, 60-day mortality and 60-90 day mortality was $1.25,1.18$ and 1.10 , respectively. In the present study, all-cause mortality was $13.9 \%$ in patients with $\mathrm{HFpEF}$, which is lower than the rates obtained in other trials (OPTIME-CHF, 27\%; ACTIV-in-CHF, 21\%) (5,19). Furthermore, hyponatremia was associated with a 2-fold increased risk of 24-month all-cause mortality. Hence, hyponatremia is a valuable predictor of prognosis in patients with HF.

A number of factors contribute to the poor outcomes in patients with HFpEF with hyponatremia. For instance, HF progression is closely linked to the activation of the neuroendocrine system, which is classically represented 


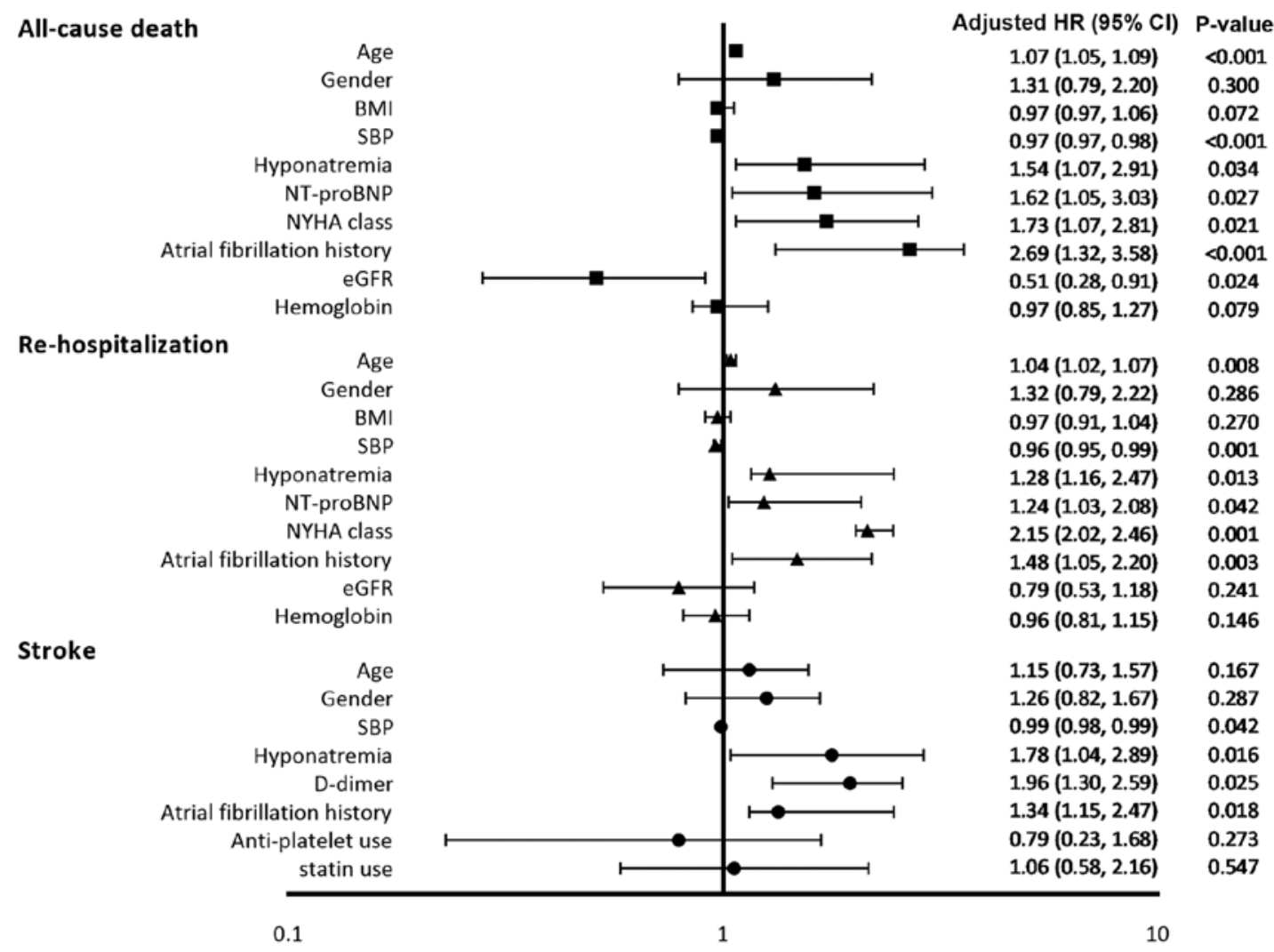

Figure 3. Predictors of all-cause mortality, re-hospitalization and stroke in patients with HFpEF. Logistic regression analysis with the Cox proportional hazard model was performed for the three endpoint events. Univariate analysis was applied prior to the multivariate analysis (data not shown). According to the results of the multivariate analysis, hyponatremia, older age, lower SBP, NT-proBNP above the median, NYHA class III-IV, a history of AF and eGFR $<60 \mathrm{ml} / \mathrm{min} / 1.73 \mathrm{~m}^{2}$ predicted higher all-cause mortality, after adjustment for sex, BMI and hemoglobin. Furthermore, hyponatremia predicted a higher re-hospitalization rate and a higher incidence of stroke. $\mathrm{HR}$, hazard ratio; $\mathrm{HFpEF}$, heart failure with preserved ejection fraction; $\mathrm{AF}$, atrial fibrillation; $\mathrm{BMI}$, body mass index; NT-proBNP, N-terminal pro B-type natriuretic peptide; NYHA, New York Heart Association; eGFR, estimated glomerular filtration rate; SBP, systolic blood pressure.

by the RAAS. Furthermore, hyponatremia may directly upregulate the activity of RAAS and arginine vasopressin (AVP), which increase the heart rate, vessel constriction and renal urine reabsorption. However, excessive activation of the neuroendocrine system may result in adverse effects, including increased cardiac afterload and myocardial oxygen consumption, cardiac cell death and myocardial fibrosis (25). However, the amount of BNP secreted into the circulation of patients with exacerbation inhibits the release of aldosterone to facilitate natriuresis $(26,27)$.

To the best of our knowledge, the present study was the first to demonstrate that hyponatremia $(\mathrm{OR}=1.78,95 \%$ $\mathrm{CI}=1.04,2.89, \mathrm{P}=0.016)$ was a stronger predictor of stroke in patients with HFpEF. Furthermore, a previous study reported that hyponatremia was associated with stroke (28). Aberrant serum sodium levels are also considered as a complication of cerebrovascular disease (29), and the possible mechanisms are associated with inappropriate secretion of antidiuretic hormone, frequent use of diuretics, as well as blood concentration and viscosity (30).

Since hyponatremia is independently associated with poor outcomes, correcting hyponatremia in a timely and appropriate manner may improve the prognosis of patients with HFpEF. There are different treatment options, the first of which includes a daily fluid allowance of 800-100 ml. As another option, the addition of furosemide with an ACEI is able to significantly improve the sodium concentration. In addition, arginine vasopressin (AVP) receptor antagonists, which have a central role in regulating water retention via these receptors, may be used. It has been revealed that anemia, NT-proBNP and NYHA cardiac function not only have an impact on the risk of mortality, but also on the incidence of hyponatremia $(14,31)$, which should be corrected as soon as possible.

The present study had several limitations. First, it was designed as an observational study and was potentially open to selection bias, as patients with severe liver disease, trauma, infection and recent surgery were excluded. Furthermore, the etiology of hyponatremia was not surveyed in the patients. The present study did not differentiate true hyponatremia from pseudo-hyponatremia and did not analyze depletion and dilution hyponatremia, but the prognosis and treatment were different between them (32). In addition, tolvaptan and AVP receptor antagonists were not widely used in the current cohort and these should be further examined to assess their effect on patients with HFpEF. Due to potential recall bias when performing the subgroup analysis, the present study did not avoid the influence of the history of atrial fibrillation on the incidence of stroke.

In conclusion, the present results indicated that hyponatremia on admission may be a useful prognostic marker for patients with HFpEF. However, larger studies are required to be performed to confirm these results, as well as to elucidate 
the mechanisms of mortality and stroke associated with hyponatremia and identify the potential benefit of correction of hyponatremia in patients with $\mathrm{HFpEF}$.

\section{Acknowledgements}

Not applicable.

\section{Funding}

This work was supported by a grant from the National Nature Science Foundation of China (grant no. 81270194).

\section{Availability of data and materials}

The datasets used and/or analyzed during the current study are available from the corresponding author on reasonable request.

\section{Authors' contributions}

Conception and design: YS, YL, CY. Clinical support and acquisition of data: YS, YL, CY. Provision of study materials or patients: YS, YL. Collection and collation of data: YS, MM, HZ, XP, XZ and FZ. Data analysis and interpretation: YS, YL. Manuscript writing: YS, MM, HZ, YL, CY. All authors read and approved the final manuscript.

\section{Ethics approval and consent to participate}

The study protocol was approved by the Research Ethical Committee of Shanghai 10th People's Hospital of Tongji University School of Medicine (Shanghai, China) and conducted according to the principles expressed in the Declaration of Helsinki. The data were retrieved from the hospital's medical record system and therefore, no additional informed consent was required. The institutional review board also waived the need for written informed consent from the participants. The privacy of patients' personal data was protected.

\section{Patient consent for publication}

Not applicable.

\section{Competing interests}

The authors declare that they have no competing interests.

\section{References}

1. Ponikowski P, Voors AA, Anker SD, Bueno H, Cleland JG, Coats AJ, Falk V, González-Juanatey JR, Harjola VP, Jankowska EA, et al: 2016 ESC guidelines for the diagnosis and treatment of acute and chronic heart failure: The task force for the diagnosis and treatment of acute and chronic heart failure of the European society of cardiology (ESC). Developed with the special contribution of the heart failure association (HFA) of the ESC. Eur J Heart Fail 18: 891-975, 2016.

2. Dunlay SM, Roger VL and Redfield MM: Epidemiology of heart failure with preserved ejection fraction. Nat Rev Cardiol 14: 591-602, 2017.

3. Ge J: Coding proposal on phenotyping heart failure with preserved ejection fraction: A practical tool for facilitating etiology-oriented therapy. Cardiol J 27: 97-98, 2020.
4. Mohan S, Gu S, Parikh A and Radhakrishnan J: Prevalence of hyponatremia and association with mortality: Results from NHANES. Am J Med 126: 1127-1137.e1, 2013.

5. Rossi J, Bayram M, Udelson JE, Lloyd-Jones D, Adams KF, Oconnor CM, Stough WG, Ouyang J, Shin DD, Orlandi C and Gheorghiade M: Improvement in hyponatremia during hospitalization for worsening heart failure is associated with improved outcomes: Insights from the acute and chronic therapeutic impact of a vasopressin antagonist in chronic heart failure (ACTIV in CHF) trial. Acute Card Care 9: 82-86, 2007.

6. Gheorghiade M, Abraham WT, Albert NM, Gattis Stough W, Greenberg BH, O'Connor CM, She L, Yancy CW, Young J and Fonarow GC; OPTIMIZE-HF Investigators and Coordinators: Relationship between admission serum sodium concentration and clinical outcomes in patients hospitalized for heart failure: An analysis from the OPTIMIZE-HF registry. Eur Heart J 28: 980-988, 2007

7. Gheorghiade M, Rossi JS, Cotts W, Shin DD, Hellkamp AS, Piña IL, Fonarow GC, DeMarco T, Pauly DF, Rogers J, et al: Characterization and prognostic value of persistent hyponatremia in patients with severe heart failure in the ESCAPE Trial. Arch Intern Med 167: 1998-2005, 2007.

8. Lu DY, Cheng HM, Cheng YL, Hsu PF, Huang WM, Guo CY, $\mathrm{Yu}$ WC, Chen $\mathrm{CH}$ and Sung SH: Hyponatremia and worsening sodium levels are associated with long-term outcome in patients hospitalized for acute heart failure. J Am Heart Assoc 5: $\mathrm{e} 002668,2016$

9. Patel YR, Kurgansky KE, Imran TF, Orkaby AR, McLean RR, Ho YL, Cho K, Gaziano JM, Djousse L, Gagnon DR and Joseph J: Prognostic significance of baseline serum sodium in heart failure with preserved ejection fraction. J Am Heart Assoc 7: e007529, 2018.

10. Park JJ, Cho YJ, Oh IY, Park HA, Lee HY, Kim KH, Yoo BS Kang SM, Baek SH, Jeon ES, et al: Short and long-term prognostic value of hyponatremia in heart failure with preserved ejection fraction versus reduced ejection fraction: An analysis of the Korean acute heart failure registry. Int J Cardiol 248: 239-245, 2017.

11. McKee PA, Castelli WP, McNamara PM and Kannel WB: The natural history of congestive heart failure: The Framingham study. N Engl J Med 285: 1441-1446, 1971.

12. Lang RM, Badano LP, Mor-Avi V, Afilalo J, Armstrong A, Ernande L, Flachskampf FA, Foster E, Goldstein SA, Kuznetsova $\mathrm{T}$, et al: Recommendations for cardiac chamber quantification by echocardiography in adults: An update from the American society of echocardiography and the European association of cardiovascular imaging. J Am Soc Echocardiogr 28: 1-39.e14, 2015.

13. Cabuk AK, Cabuk G, Sayin A, Karamanlioglu M, Kilicaslan B, Ekmekci C, Solmaz H, Aslanturk OF and Ozdogan O: Do we overestimate left ventricular ejection fraction by two-dimensional echocardiography in patients with left bundle branch block? Echocardiography 35: 148-152, 2018.

14. Bavishi C, Ather S, Bambhroliya A, Jneid H, Virani SS, Bozkurt B and Deswal A: Prognostic significance of hyponatremia among ambulatory patients with heart failure and preserved and reduced ejection fractions. Am J Cardiol 113: 1834-1838, 2014.

15. Kusaka H, Sugiyama S, Yamamoto E, Akiyama E, Matsuzawa Y, Hirata Y, Fujisue K, Kurokawa H, Matsubara J, Sugamura K, et al: Low-normal serum sodium and heart failure-related events in patients with heart failure with preserved left ventricular ejection fraction. Circ J 80: 411-417, 2016.

16. Rusinaru D, Buiciuc O, Leborgne L, Slama M, Massy Z and Tribouilloy C: Relation of serum sodium level to long-term outcome after a first hospitalization for heart failure with preserved ejection fraction. Am J Cardiol 103: 405-410, 2009.

17. Upadhyay A, Jaber BL and Madias NE: Incidence and prevalence of hyponatremia. Am J Med 119 (7 Suppl 1): S30-S35, 2006.

18. Gheorghiade M, Gattis WA, O'Connor CM, Adams KF Jr, Elkayam U, Barbagelata A, Ghali JK, Benza RL, McGrew FA, Klapholz M, et al: Effects of tolvaptan, a vasopressin antagonist, in patients hospitalized with worsening heart failure: A randomized controlled trial. JAMA 291: 1963-1971, 2004.

19. Klein L, O'Connor CM, Leimberger JD, Gattis-Stough W, Piña IL, Felker GM, Adams KF Jr, Califf RM and Gheorghiade M; OPTIME-CHF Investigators: Lower serum sodium is associated with increased short-term mortality in hospitalized patients with worsening heart failure: Results from the outcomes of a prospective trial of intravenous milrinone for exacerbations of chronic heart failure (OPTIME-CHF) study. Circulation 111: 2454-2460, 2005 . 
20. Stolfo D, Uijl A, Vedin O, Strömberg A, Faxén UL, Rosano GMC, Sinagra G, Dahlström U and Savarese G: Sex-based differences in heart failure across the ejection fraction spectrum: Phenotyping, and prognostic and therapeutic implications. JACC Heart Fail 7: 505-515, 2019.

21. Baldasseroni S, Urso R, Orso F, Bianchini BP, Carbonieri E, Cirò A, Gonzini L, Leonardi G, Marchionni N and Maggioni AP; IN-CHF Investigators: Relation between serum sodium levels and prognosis in outpatients with chronic heart failure: Neutral effect of treatment with beta-blockers and angiotensin-converting enzyme inhibitors: Data from the Italian network on congestive heart failure (IN-CHF database). J Cardiovasc Med (Hagerstown) 12: 723-731, 2011.

22. Lee WH and Packer M: Prognostic importance of serum sodium concentration and its modification by converting-enzyme inhibition in patients with severe chronic heart failure. Circulation 73 257-267, 1986.

23. Yoo BS, Park JJ, Choi DJ, Kang SM, Hwang JJ, Lin SJ, Wen MS, Zhang J and Ge J; COAST investigators: Prognostic value of hyponatremia in heart failure patients: An analysis of the clinical characteristics and outcomes in the relation with serum sodium level in asian patients hospitalized for heart failure (COAST) study. Korean J Intern Med 30: 460-470, 2015.

24. Abraham WT, Fonarow GC, Albert NM, Stough WG, Gheorghiade M, Greenberg BH, O'Connor CM, Sun JL, Yancy CW and Young JB; OPTIMIZE-HF Investigators and Coordinators: Predictors of in-hospital mortality in patients hospitalized for heart failure: Insights from the organized program to initiate lifesaving treatment in hospitalized patients with heart failure (OPTIMIZE-HF). J Am Coll Cardiol 52: 347-356, 2008.

25. Vergaro G, Aimo A, Prontera C, Ghionzoli N, Arzilli C, Zyw L, Taddei C, Gabutti A, Poletti R, Giannoni A, et al: Sympathetic and renin-angiotensin-aldosterone system activation in heart failure with preserved, mid-range and reduced ejection fraction. Int J Cardiol 296: 91-97, 2019.
26. Azim A and Jena S: BNP: Is it an ideal marker to assess volume status in patients with hyponatremia and natriuresis? Neurol India 66: 1392-1393, 2018

27. Tobin G, Chacko AG and Simon R: Evaluation of NT-ProBNP as a marker of the volume status of neurosurgical patients developing hyponatremia and natriuresis: A pilot study. Neurol India 66: 1383-1388, 2018.

28. Wannamethee G, Whincup PH, Shaper AG and Lever AF: Serum sodium concentration and risk of stroke in middle-aged males. J Hypertens 12: 971-979, 1994.

29. Zheng B, Qiu Y, Jin H, Wang L, Chen X, Shi C and Zhao S: A predictive value of hyponatremia for poor outcome and cerebral infarction in high-grade aneurysmal subarachnoid haemorrhage patients. J Neurol Neurosurg Psychiatry 82: 213-217, 2011.

30. Writing Group Members, Mozaffarian D, Benjamin EJ, Go AS, Arnett DK, Blaha MJ, Cushman M, Das SR, de Ferranti S, Després JP, et al: Heart disease and stroke statistics-2016 update: A report from the American heart association. Circulation 133: e38-e360, 2016.

31. McGirt MJ, Blessing R, Nimjee SM, Friedman AH Alexander MJ, Laskowitz DT and Lynch JR: Correlation of serum brain natriuretic peptide with hyponatremia and delayed ischemic neurological deficits after subarachnoid hemorrhage. Neurosurgery 54: 1369-1373; discussion 1373-1364, 2004.

32. Verbrugge FH, Steels P, Grieten L, Nijst P, Tang WH and Mullens W: Hyponatremia in acute decompensated heart failure: Depletion versus dilution. J Am Coll Cardiol 65: 480-492, 2015.

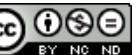

This work is licensed under a Creative Commons Attribution-NonCommercial-NoDerivatives 4.0 International (CC BY-NC-ND 4.0) License. 